\title{
Reproducibility of Human Brain Activity Following Repeated Oesophageal Stimulation
}

\author{
S. J. Coen ${ }^{1}$, L. J. Gregory ${ }^{2}$, L. Yágüez ${ }^{1}$, D. Hall ${ }^{2}$, E. Amaro ${ }^{1}$, S. Smale ${ }^{3}$, S. C. Williams ${ }^{1}$, D. G. Thompson ${ }^{2}$, Q. Aziz ${ }^{2}$ \\ ${ }^{1}$ Institute of Psychiatry, London, United Kingdom, ${ }^{2}$ University of Manchester, Manchester, United Kingdom, ${ }^{3}$ Kings College Hospital, London, United Kingdom
}

\section{Synopsis}

Despite the recent growth in studies on the central processing of visceral sensations, the test-retest reliability of the neural correlates of visceral sensation remain unclear. In order to test the variance between scan sessions, at the group level, volunteers underwent a modified box-car functional magnetic resonance imaging (fMRI) experiment, involving periods of non-painful and painful oesophageal stimulation on three separate occasions. Painful stimulation produced robust activation in many cerebral regions previously associated with visceral processing. Non-painful stimulation activated a similar network but showed greater variability between scans.

\section{Introduction}

Previous studies have identified various cerebral regions involved in the processing of visceral sensations (1). It is often assumed that activations obtained during these studies are inherently reliable, and therefore the conclusions drawn are based on this assumption. In fact, the cerebral activation during fMRI sessions can be influenced by many variables including scanner noise, subject movement and cognition, such as habituation, attention and learning. While the cerebral processing of visceral sensation has been studied, information regarding test-retest reliability of fMRI studies applied to this area of research remains unclear.

Aims: The purpose of this study was to assess the reproducibility of the neural correlates of oesophageal sensation, using fMRI.

\section{Methods}

Subjects: 7 healthy volunteers ( 5 male, mean age 22 years, \pm 7 months) participated in the study. All subjects gave informed, written consent prior to intubation and scanning. The study was approved by the local ethics committee for research.

Oesophageal stimulation: a standard manometry catheter with a silicone balloon attached was passed trans-nasally into the lower oesophagus (35cm from the nostril). The catheter was attached to a pump that inflated the balloon with air at regular intervals. The experiment examined two conditions; non-painful, (50\% of the difference between sensory and pain thresholds), and painful (pain threshold) stimulation intensities, using phasic balloon distension in the distal oesophagus. The order of presentation of these intensities was counterbalanced across scans. A modified block design was employed for each intensity, where "active" and "rest" phases were repeated five times. Behavioural data measuring the subjective perception of the stimulus $(0=$ non-painful, $5=$ discomfort, $10=$ extreme pain) were also acquired after each active epoch, using visual analogue scales (VAS). This experimental procedure was repeated on two additional occasions (three scans in total) to investigate the consistency of cerebral activation over time. fMRI acquisition: Functional Magnetic Resonance Imaging was performed using a GE Neuro-optimised 1.5 Tesla system (General Electric, Milwaukee WI, USA), based at the Maudsley Hospital, London. Sixteen $7 \mathrm{~mm}$ slices (0.7 $\mathrm{mm}$ gap) parallel to the bicomissural plane were acquired, with a repetition time (TR) of 3 seconds and an echo time (TE) of 40ms, flip angle $90^{\circ}$. A total of 122 T2* weighted images per slice, depicting BOLD contrast (Ogawa et al, 1990) were collected over a six minute and six second period of continuous acquisition during which, subjects received localised phasic distensions to the oesophagus.

Image Analysis: Spatially realigned BOLD responses were modelled as the weighted sum of the input function convolved with two Poisson functions. A goodness of fit statistic was computed and a voxel-wise inference was carried out non-parametrically. At the group level, individual statistic maps were transformed into standard stereotactic space and median activation images constructed.

\section{Results}

Analysis of mean VAS ratings for painful stimulation revealed a significant $\left(\chi^{2}=27.67\right.$, df $\left.2, \mathrm{P}=0.001\right)$ progressive decrease in perceived intensity of stimulus from the first scanning occasion to the third scanning occasion ( mean VAS $( \pm$ SEM); scan $1=7.3 \pm 0.45$, scan $2=6.1 \pm 0.86$, scan $3=$ $4.8 \pm 0.81$ ), which was not seen following non-painful stimulation.

In response to painful stimulation, highly reproducible activation was seen in the anterior cingulate gyrus (ACG) (BA24 \& 32 ), bilateral insula, supplementary motor area (SMA), thalamus, bilateral primary and secondary sensory cortices (SI\&SII) and dorsolateral prefrontal cortex. Further analysis of whole brain activation, revealed a significant $(\mathrm{P}<0.05$, Bonferroni corrected) progressive decrease in cerebral activity, from the first scan to the last, in the anterior cingulate (ACG), SMA and SI, which was not found in any other region. Activation in response to non-painful stimulation was observed in similar regions to those seen during painful stimulation, but with greater variability between scanning occasions.

\section{Discussion}

Painful stimulation of the oesophagus produces robust activation in many brain regions previously associated with visceral pain. During painful stimulation, BOLD contrast in the anterior cingulate, SMA and SI decreased from the first to the final scan. This may be due to habituation of the stimulus, however the analogous decrease in VAS ratings, and the implication that the anterior cingulate and SI encode stimulus intensity during somatic stimulation (2), suggest that the decrease is more likely to be a result of lower stimulation levels. The fact that VAS ratings progressively decreased between scans, may due to adaptation of the oesophagus or perhaps modulation of pain perception by anxiety (3), which is greater during visceral than somatic stimulation (4). Anxiety, we suggest, would decrease at each scanning occasion as the paradigm and environment becomes more predictable and less threatening, resulting in lowered pain ratings. Non-painful stimulation results in a similar pattern of cerebral activation, but is more variable between scans, suggesting that a non-painful stimulation level is more susceptible to changes in levels of attention, arousal and anxiety. This phenomenon may influence neural activity and therefore have an effect on reproducibility, as well as having considerable implications for future studies requiring serial functional imaging assessment.

\section{$\underline{\text { References }}$}

1. Aziz Q, Thompson DG. Brain-Gut Axis in Health and Disease. Gastroenterology 1998; 114: 559-578

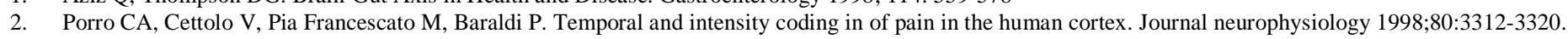

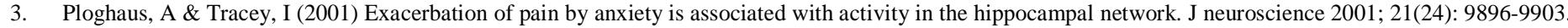

4. Strigo IA, Bushnell MC, Boivin M, Duncan GH. Psychophysical analysis of visceral and cutaneous pain in human subjects. Pain 2002; 97: 235-246. 